\title{
Swedish snuff and incidence of cardiovascular disease. A population-based cohort study Ellis Janzon ${ }^{\dagger 1}$ and Bo Hedblad ${ }^{*+2}$
}

Address: ${ }^{1}$ Department of Health and Society, Malmö University, Malmö, Sweden and ${ }^{2}$ Department of Clinical Science, Research Groups of Cardiovascular Epidemiology Lund University, Malmö University Hospital, Malmö, Sweden

Email: Ellis Janzon - ellis.janzon@mah.se; Bo Hedblad* - bo.hedblad@med.lu.se

* Corresponding author †Equal contributors

Published: 27 May 2009

BMC Cardiovascular Disorders 2009, 9:21 doi:| 0.| |86/|47|-226|-9-2|
Received: 30 September 2008

Accepted: 27 May 2009

This article is available from: http://www.biomedcentral.com/|47/-226I/9/2 I

(c) 2009 Janzon and Hedblad; licensee BioMed Central Ltd.

This is an Open Access article distributed under the terms of the Creative Commons Attribution License (http://creativecommons.org/licenses/by/2.0), which permits unrestricted use, distribution, and reproduction in any medium, provided the original work is properly cited.

\begin{abstract}
Background: The relationship between smoking and an increased incidence of cardiovascular diseases is well known. Whether smokeless tobacco (snuff) is related to myocardial infarction (MI) or stroke is still controversial. Aim of this study was to explore whether snuff users have an increased incidence of MI or stroke.
\end{abstract}

Methods: A total of 16754 women and 10473 men (aged 45-73 years), without history of cardiovascular disease (CVD), belonging to the population-based "Malmö Diet and Cancer" study were examined. Incidence of $\mathrm{Ml}$ and stroke were monitored over 10.3 years.

Results: Snuff was used by 737 (7.0\%) men and 75 (0.4\%) women, respectively. Among men, snuff was significantly associated with low occupation level, single civil status, high BMI and with current and former smoking. In women, snuff was associated with lower systolic blood pressure. A total of 964 individuals (3.5\%), i.e.544 men (5.3\%) and 420 (2.5\%) women suffered a MI during the followup period. The corresponding numbers of incident stroke cases were 1048, i.e. 553 men (5.3\%) and $495(3.0 \%)$ women, respectively. Snuff was not associated with any statistically significant increased risk of $\mathrm{Ml}$ or stroke in men or women. The relative risks (RR) in male snuff users compared to nonusers were 1.05 (95\% confidence interval $(\mathrm{Cl}): 0.8-\mathrm{I} .4, \mathrm{p}=0.740)$ for incident $\mathrm{MI}$ and $0.97(0.7-$ I.4, $\mathrm{p}=0.878$ ) for stroke, after taking age and potential confounders into account. In women none of the $420(2.5 \%)$ women who were snuff users had a $\mathrm{Ml}$ and only one suffered a stroke during the follow-up.

Conclusion: Several life-style risk factors were more prevalent in snuff-users than in non-users. However, the present study does not support any relationship between snuff and incidence of cardiovascular disease in men.

\section{Background}

The relationship between smoking and an increased incidence of cardiovascular diseases (CVD) is well known. Whether smokeless tobacco, in this study defined as snuff, is related to incidence of CVD is less clear. Lately several studies have explored the relationships between snuff and CVD, and the results are not consistent [1-13]. In an earlier study of construction workers, a significantly increased risk of MI was reported among snuff users $[7,8]$. Snuff users also had a significantly higher prevalence of 
hypertension in that study [8]. In contrast, several other studies from the general population found no relationship between snuff and incidence of MI $[2-5,10,12,13]$. Some scientists have highlighted the need for more studies on snuff and incidence of CVD[1,2]. The purpose of this population based cohort study was to explore whether snuff users have an increased incidence of myocardial infarction (MI) and stroke.

\section{Methods \\ Subjects}

The Malmö Diet and Cancer (MDC) cohort was used to examine snuff use and its effect on incidence of CVD. All subjects who were 45-73 years old, and who lived in the city of Malmö, Sweden were invited. The health examination was performed at the Malmö University Hospital from March 1991 to February 1996. The subjects were invited by letters and advertisement in newspapers. Of the eligible population of approximately 70000 citizens, 28 449 subjects, i.e. 17203 women and 11246 men, respectively participated. The participation rate was approximately $40 \%$ [14,15]. The MDC cohort and the characteristics of the non-participants have been described previously [15]. The representativeness of the MDC study has been evaluated by a comparison with subjects, in corresponding age groups, who participated in 1994 in a mailed questionnaire health survey in the city of Malmö $[15,16]$. In that survey, non attendance was associated with a lower prevalence of smoking and a higher rate of mortality. However, prevalence of smoking and obesity in the MDC was rather similar compared to the mailed health study (prevalence of smoking 39.9\% vs. $37.5 \%$ and $12.4 \%$ vs. $11.8 \%$ for prevalence of obesity, respectively).

After exclusion of subjects with a history of MI or stroke, and subjects with missing information about BMI, blood pressure, diabetes or tobacco habits, the cohort consisted of 27227 individuals, 16754 women and 10473 men, respectively. Mean age was $57.4+7.6$ years in women and $59.1+7.0$ years in men.

\section{Screening examination}

Measurements of height (in $\mathrm{cm}$ ), weight (in kilograms) and blood pressure (in $\mathrm{mmHg}$ ) were performed. The participants also completed a comprehensive self-administered questionnaire about life style habits and medical history and treatment [14].

\section{Smoking habits and use of snuff}

Smoking was assessed in a self-administered questionnaire, and the subjects were categorized as never smokers, ex-smokers and current smokers (regular or occasional smokers). Daily cigarette consumption among current smokers was assessed by the questions "how many cigarettes do you smoke per day"?

Snuff use was assessed by the question "Do you use snuff?" In subjects confirming the use of snuff the weekly consumption of snuff was obtained by the question "How many packages of snuff do you consume every week?" We dichotomized weekly snuff consumption into three groups: a) 1-2 packages per week as low consumption, b) 3-5 packages per week as medium consumption and c) more than six packages per week as high consumption.

\section{Chewing tobacco and use of nicotine gum}

The habits of tobacco chewing and using nicotine gum were obtained by the questions: " "Do you chew tobacco" and "Do you use nicotine chewing gum?"

\section{Hypertension}

Blood pressure was measured twice in the supine position after 10 minutes rest using a mercury sphygmomanometer. Subjects who had systolic blood pressure (SBP) _ 140 $\mathrm{Mm} / \mathrm{Hg}$ or diastolic blood pressure (DBP) _ $90 \mathrm{mmHg}$ or blood pressure-lowering drug treatment of hypertension were considered to have hypertension [17].

\section{Diabetes mellitus}

Participants who reported that they had diabetes or who used anti-diabetic medication was considered to have diabetes. As fasting blood glucose and lipids were available only on subjects belonging to the cardiovascular project ( $\mathrm{n}=5$ 500) [18], It was not possible to define diabetes on all study subjects according to present or previous recommendations for classification of diabetes mellitus [19].

\section{Body mass index (BMI)}

BMI was calculated as weight $/$ height ${ }^{2}\left(\mathrm{~kg} / \mathrm{m}^{2}\right)$.

\section{Physical activity}

Physical activity during leisure time was revealed through 18 questions covering a range of activities in the four seasons. The number of minutes per week for each activity was multiplied by an intensity coefficient, and an overall leisure time physical activity scores was created. Scores were divided into four quartiles and subsequently categorized as a) low (quartile 1), b) moderate (quartile 2 to 3 ), and c) high (quartile 4) levels [20].

\section{Information on socio-economic circumstances}

Information on occupation and marital status was assessed by the questionnaire. The categorization into occupational groups based on the Swedish socio-economic index (SEI) [21] has been presented previously $[22,23]$. This classification takes into consideration the educational level needed for the job, the level of responsibility in the work organization as well as the actual work 
task [22]. In this study data were dichotomized into four groups only, due to few cases. High and medium levels non-manual employees was dichotomized into the same group as "High occupational level" (SEI groups 46-69)", and contained the following groups: high level (i.e., business executives, university-engineers and teachers) and medium level employees (i.e., reg. nurses, employees, computer operators and high school teachers).

Low-level non-manual employees (SEI groups 33-36, i.e. office assistants, sales staff and secretaries, etc) were categorized as "Medium occupation level".

Skilled manual workers, unskilled workers and unspecified occupational groups (i.e. early retired women, housewives, students and unemployed) (SEI groups 11-22) were categorized as "low level occupational". The group "others" contained self employed* and farmers i.e. (SEI groups 70-99) [22,23].

\section{Marital status}

The questionnaire had the following four categories: Married, unmarried, divorced and widowed. In accordance with previous studies we dichotomized the cohort into the status living alone or not [22].

\section{Education}

Education level was assessed in the questionnaire as previously described and divided into low (9 years education or less) and high education level (at least secondary graduation). The dichotomized variable education was used in the analyses [22].

\section{Incidence of stroke and MI}

Each participant was followed from the baseline examination until the first incident MI or stroke event, death, emigration out of Sweden, or Dec 31, 2004, whichever came first.

Incident stroke was defined as ICD-9 codes 430, 431, 434 and $436[24,25]$. MI was defined as nonfatal MI (ICD-9 code 410) or fatal ischemic heart disease (ICD-9 code 410-414). The National hospital discharge register, the Stroke register of Malmö [26] and the Swedish hospital discharge register were used for case retrieval.

\section{Statistics}

The Cox proportional hazards model was used to assess the relationship between snuff use and incidence of MI and stroke, respectively. The Cox model was also used to adjust the relationships for other risk factors. One way analyses of variance and logistic regression was used to compare risk factor in users and non-users of snuff. A general linear model was used to adjust the mean values (i.e., blood pressure and BMI) for other risk factors

\section{Results}

Snuff was used by a total of 812 individuals, i.e. 737 $(7.0 \%)$ men and $75(0.4 \%)$ women, respectively. Of those subjects $764(94.1 \%)$ reported weekly snuff packages consumption. Among men 54.5\% ( $\mathrm{n}=402)$ were categorized as low, $32.2 \%(n=237)$ as medium and $9.0 \%(n=66)$ as high consumers. The corresponding figures among women were $58.7 \%(\mathrm{n}=44), 14.7 \%(\mathrm{n}=11)$ and $0.1 \%(\mathrm{n}$ $=4$ ), respectively.

Chewing tobacco was unusual in this cohort, being reported by only 96 persons $(0.4 \%)$. Nicotine gum was used by a total of 497 individuals $(1.8 \%)$, i.e. 131 (26.4\%) men and 364 (73.6\%) women, respectively data not presented in tables.

\section{Snuff, smoking habits and cardiovascular risk factors}

The distribution of risk factors in users and non-users of snuff is presented in Table 1. Mean age for snuff users was significantly lower in both men and women compared to non-users. Smoking and former smoking was more common in snuff-users compared to non-users. However, the mean daily cigarette consumption (in grams) in snuff users compared to non-users was significantly lower both among male and female current smokers. Former smoking was also higher among snuffusers compared to non-users, significantly only for men. Very few never smokers reported use of snuff (i.e. 67 men and 27 women). BMI was slightly higher in male snuff users, and this relationship remained significant after adjustment for age [adjusted means \pm standard error of the mean; $26.2 \pm 0.035$ vs. $\left.26.6 \pm 0.013 \mathrm{~kg} / \mathrm{m}^{2}\right]$. In men, blood pressure was rather similar in snuff users and nonusers, however, prevalence of blood pressure lowering treatment was significantly lower in male snuff-users (i.e. $14.9 \%$ vs. $18.5 \%, p=0.016$ ) [Table 1]. Female snuff users had statistically significant lower systolic blood pressure and prevalence of hypertension than non-users. These relationships were markedly attenuated and became non-significant after taking age and BMI into account (data not shown) In addition to that, fewer male snuff users than non-users were currently on treatment with blood pressure lowering agents (i.e. $14.9 \%$ vs. $18.5 \%, \mathrm{p}=0.016$ for men, and $9.3 \%$ vs. $15.7 \%, \mathrm{p}=0.129$ for women [Table 1].

\section{Snuff and socio-demographic risk factors}

Significantly more male snuff users than non-users were living alone and categorized as belonging to the lowlevel occupational group. Among women, however, the proportion that was categorized as belonging to lowlevel occupational group was lower among snuff users compared to non-users $25.2 \%$ vs. $44.3 \%, p=0.122$ [Table 1]. 
Table I: Distributions of risk factors in 27227 individuals in relation to smokeless tobacco (snuff)

\begin{tabular}{|c|c|c|c|c|c|c|}
\hline & \multicolumn{3}{|l|}{ Men $(n=10473)$} & \multicolumn{3}{|c|}{ Women $(n=16754)$} \\
\hline & $\begin{array}{l}\text { Snuff non users } \\
(n=9736)\end{array}$ & Snuff users $(n=737)$ & $P$ value & $\begin{array}{l}\text { Snuff non users } \\
(\mathrm{n}=16679)\end{array}$ & Snuff users $(n=75)$ & $P$ value \\
\hline Age (years) & $59.2 \pm 7.6$ & $56.8 \pm 6.7$ & $<0.001$ & $57.4 \pm 7.9$ & $54.6 \pm 7.2$ & 0.002 \\
\hline \multicolumn{7}{|l|}{$\begin{array}{l}\text { Blood pressure } \\
(\mathrm{BP}, \mathrm{mm} \mathrm{Hg})\end{array}$} \\
\hline Systolic & $143.9 \pm 19.3$ & $143.0 \pm 19.3$ & 0.227 & $139.2 \pm 20.2$ & $134.4 \pm 21.2$ & 0.040 \\
\hline Diastolic & $88.0 \pm 9.8$ & $88.2 \pm 9.9$ & 0.587 & $84.0 \pm 9.7$ & $82.0 \pm 10.4$ & 0.090 \\
\hline Hypertension, \% (n) & $65.2(6351)$ & $65.8(485)$ & 0.708 & $53.2(8872)$ & $37.3(28)$ & 0.006 \\
\hline $\begin{array}{l}\text { Use of BP lowering } \\
\text { treatment, \% (n) }\end{array}$ & & $14.9(110)$ & 0.016 & $15.7(2623)$ & $9.3(7)$ & 0.129 \\
\hline $\mathrm{BMI}(\mathrm{kg} / \mathrm{m} 2)$ & $26.2 \pm 3.5$ & $26.6 \pm 3.5$ & 0.004 & $25.4 \pm 4.2$ & $25.1 \pm 3.9$ & 0.528 \\
\hline Diabetes mellitus \%(n) & $3.9(368)$ & $3.9(29)$ & 0.832 & $2.4(398)$ & $\mathrm{I} .3(\mathrm{I})$ & 0.551 \\
\hline \multicolumn{7}{|l|}{ Smoking habits } \\
\hline Never smoker, \% (n) & $30.3(2946)$ & $9.1(67)$ & 0.000 & $44.4(7404)$ & $36.0(27)$ & 0.144 \\
\hline Ex-smoker, \% (n) & $41.2(4014)$ & $57.0(420)$ & 0.000 & $27.6(4599)$ & $36.0(27)$ & 0.103 \\
\hline Current smoker,\%(n) & $28.5(2776)$ & $33.9(250)$ & 0.002 & $28.0(4676)$ & $28.0(21)$ & 0.995 \\
\hline $\begin{array}{l}\text { Daily (grs) cigarette } \\
\text { consumption by current } \\
\text { smokers }\end{array}$ & $16.1 \pm 9.0$ & $12.3 \pm 9.7$ & 0.001 & $12.9 \pm 7.1$ & $7.8 \pm 6.4$ & 0.001 \\
\hline \multicolumn{7}{|l|}{ Occupational status } \\
\hline Low level, \% (n) & $45.4(3866)$ & $57.0(369)$ & 0.000 & $44.3(6522)$ & $25.2(25)$ & 0.122 \\
\hline Medium level, \% (n) & $18.0(1532)$ & I $3.8(89)$ & 0.007 & $20.0(2944)$ & $23.9(17)$ & 0.409 \\
\hline High level, \% (n) & $14.5(1234)$ & II.3 (73) & 0.025 & $14.2(2092)$ & $22.5(16)$ & 0.460 \\
\hline Others, \% (n) & $22.2(1889)$ & $17.9(116)$ & 0.212 & $21.4(315 I)$ & $18.3(13)$ & 0.524 \\
\hline \multicolumn{7}{|l|}{ Civil status } \\
\hline Single, \% (n) & $27.0(2630)$ & $32.6(240)$ & 0.001 & $39.2(6545)$ & $46.7(35)$ & 0.117 \\
\hline
\end{tabular}

Values are shown as mean \pm SD unless otherwise stated. For occupational status percentages are of numbers classifiable

\section{Incidence of cardiovascular disease}

During the follow-up period a total of $544(5.3 \%)$ men had MI and 553 (5.3\%) had stroke. The corresponding figures for women were $420(2.5 \%)$ for MI and 495 $(3.0 \%)$ for stroke (data not shown I tables).

In men, snuff use was not significantly related to risk of incident MI during the follow-up period, whether adjustment was made for age only (relative risk, $\mathrm{RR}=0.89,95 \%$ confidence interval CI: 0.7-1.2), or when taking other risk factors into account (RR: 1.05; 95\% CI: 0.8-1.4 [Table 2].

In men who used snuff, the age-adjusted RR for stroke was $0.88,95 \%$ CI: $0.6-1.2$. This RR remained quite similar after taking other risk factors into account (RR: 0.97; 95\% CI: 0.7-1.4 [Table 2]. No significant relationship between snuff and incidence of MI or stroke was observed in any of the categories of smoking. However, the number of MI and stroke were very small among snuff users who never had smoked ( $\mathrm{n}=4$ and $\mathrm{n}=4$, respectively).

None of the 420 women who suffered a MI were snuff users. Furthermore, there was only one stroke case among female snuff users. As the number of events was based on only one case no further statistical analysis was performed on this matter among women.

The age-adjusted RR for incident MI was 1.5 times higher (95\% CI: 0.5-4.5, p = 0.512) in men who chew tobacco than in men who used snuff. After further adjustment for other risk factors it was even higher (RR: 1.7, 95\% CI: 0.5$5.2, \mathrm{p}=0.368$, data not shown in tables). The RR for MI in tobacco chewing women was not evaluated due to small numbers.

\section{Discussion}

It is well known, and well accepted, that tobacco smoke has many negative health effects $[27,28]$. The effects of smokeless tobacco, snuff, on health are less well explored, though lately more studies have been published [1-13,2931]. Fewer studies have explored the association between snuff and CVD, and to our knowledge, there are only few studies on women $[3,10]$. During the 10 -year follow-up only one of the 75 female snuff users suffered a stroke and none a MI. Because of these small numbers in females, our analyses are restricted to males. The present study does not support the hypothesis that snuff is a risk factor for incident myocardial infarction or stroke in men. We 
Table 2: Incidence of and RR $(95 \% \mathrm{CI})$ for $\mathrm{MI}$ and stroke in relation to snuff in men

\begin{tabular}{|c|c|c|c|c|}
\hline & & \multicolumn{3}{|c|}{ Smokeless tobacco (snuff) } \\
\hline & & No $N=9736$ & Yes $N=737$ & $P$ value \\
\hline \multirow[t]{4}{*}{ MI n (\%) } & All & $5 I I(5.2)$ & $33(4.5)$ & \\
\hline & Never smokers & II 4 (3.9) & $4(6.0)$ & \\
\hline & Ex-smokers & $180(4.5)$ & $15(3.6)$ & \\
\hline & Smokers & $217(7.8)$ & $14(5.6)$ & \\
\hline \multirow[t]{4}{*}{ Age-adjusted RR } & All & 1.0 & $0.89(0.7-\mid .2)$ & 0.408 \\
\hline & Never smokers & 1.0 & $0.61(0.2-1.5)$ & 0.280 \\
\hline & Ex-smokers & 1.0 & $0.79(0.5-1.2)$ & 0.251 \\
\hline & Smokers & 1.0 & $1.2(0.8-1.9)$ & 0.393 \\
\hline \multirow[t]{4}{*}{ Risk factor adjusted* } & All & 1.0 & $1.05(0.8-1.4)$ & 0.740 \\
\hline & Never smokers & 1.0 & $0.75(0.3-1.8)$ & 0.532 \\
\hline & Ex-smokers & 1.0 & $0.81(0.5-1.2)$ & 0.304 \\
\hline & Smokers & 1.0 & $1.31(0.8-2.0)$ & 0.236 \\
\hline \multirow[t]{4}{*}{ Stroke n (\%) } & All & $518(5.3)$ & $35(3.5)$ & \\
\hline & Never smokers & $136(4.6)$ & $4(6.0)$ & \\
\hline & Ex-smokers & $211(5.3)$ & $18(4.3)$ & \\
\hline & Smokers & $17 \mid(6.2)$ & $13(5.2)$ & \\
\hline \multirow[t]{4}{*}{ Age-adjusted RR } & All & 1.0 & $0.88(0.6-1.2)$ & 0.470 \\
\hline & Never smokers & 1.0 & $0.54(0.2-1.8)$ & 0.226 \\
\hline & Ex-smokers & 1.0 & $0.9 \mid(0.6-1.5)$ & 0.719 \\
\hline & Smokers & 1.0 & $1.03(0.6-1.8)$ & 0.929 \\
\hline \multirow[t]{4}{*}{ Risk factor adjusted* } & All & 1.0 & $0.97(0.7-1.4)$ & 0.878 \\
\hline & Never smokers & 1.0 & $0.59(0.2-1.5)$ & 0.311 \\
\hline & Ex-smokers & 1.0 & $0.88(0.5-1.4)$ & 0.632 \\
\hline & Smokers & 1.0 & $1.13(0.6-2.0)$ & 0.663 \\
\hline
\end{tabular}

* adjusted for age, BMI, smoking habits, diabetes mellitus, hypertension, physical activity, marital status and occupation, RR, relative risk and $95 \%$ confidence interval.

also could not confirm previously reported findings that snuff users have higher blood pressure than non-users [8].

Snuff users generally had lower occupation level, among men, and were more often single and current or former smokers than non-users of snuff. BMI was somewhat higher in male snuff users. There was, however, no indication of any significant relationship between snuff and an increased prevalence of hypertension or diabetes. Mean blood pressure showed no statistically significant difference between male or female snuff users and non-users after taking age and BMI into account. The absence of a relationship between snuff use and high blood pressure is in accordance with results from other studies discussed in a review study by Lee 2007 [5] but in contrast to a study of construction workers [8].

Both smoking and smokeless tobacco have been associated with lower weight [31]. In this study male snuff users had higher BMI than non-users, which remained after adjustment for age and smoking habits. No difference was observed in women. Because snuff often is used by smokers who try to quit, and smoking cessation is associated with weight gain, this could be one explanation for the difference in BMI in men. It is well known that women often use smoking to control weight, but weight regulation does not seem to be a common reason for using smokeless tobacco $[32,33]$.

One question is whether the statistical power was adequate to detect a relationship between snuff and incidence of CVD. Bolinder et al [8] reported that the age-adjusted RR of CVD was approximately 1.4 (95\% CI: $1.2-1.6)$ for snuff users compared to non-users. If the risk associated with snuff had been of that magnitude, our probability of detecting a significant difference in incidence of MI would be about $75 \%$. The statistical power for a combined endpoint of MI and stroke in the present study would be about $90 \%$. We therefore believe that the statistical power of our study was adequate.

The MDC cohort was used in the present study. The participation rate was low in this cohort, approximately $40 \%$ [15]. However, when comparing the present cohort with results from a health survey based on a mailed questionnaire to subjects living in the city of Malmö 1994, in which the participation rates were $80 \%$, it has been shown that the present cohort is fairly representative with respect to e.g., smoking habits and BMI [16]. The mortality rates 
in the study by Manjer et al were, however, higher among non-participants.

The results from the present study are similar to what has been reported from other population-based studies [2$4,6,10,12,13]$ and in contrast to some other Swedish studies $[7,8]$. As use of snuff historically has been closely associated to inferior socioeconomic position, it cannot be ruled out that the differences between the present study and the results of males by Bolinder et al [8] could be explained by differences in study populations and time periods. Ramstrom et al, [29] stated that people who stop smoking often start using snuff and reported that 58\% used snuff as an aid to stop smoking. Furthermore, many continued to use snuff after smoking cessation. In the study by Huhtasaari et al, $50 \%$ of snuff users were former smokers [12]. In this present study we also found $57 \%$ snuff users among male ex-smokers. This fact sometimes causes frustration in caregivers and public health professionals. Whether snuff should be used as a device to stop smoking is intensively debated in Sweden. On one hand, there is no doubt that the health hazard of snuff is considerably smaller than that of smoking. On the other hand, there are several other nicotine based products for tobacco cessation and we cannot exclude the possibility that snuff could increase the risk of cancer $[27,28]$. In our opinion, more studies are needed to fully clarify the effects of snuff, especially in women, during pregnancy and in older individuals. It is important that information about snuff given to patients and the general public is accurate and reliable. All findings should be reported in order to increase the knowledge about the health effects of snuff.

\section{Conclusion}

Even though some life-style risk factors seem to be more prevalent in snuff-users, the present study does not support any independent relationship between snuff and incidence of MI or stroke in men.

\section{Competing interests}

The authors declare that they have no competing interests.

\section{Authors' contributions}

EJ participated in the design of the study, performed the statistical analysis and drafted the manuscript. BH conceived of the study, participated in its design and revised the manuscript critically. All authors read and approved the final manuscript.

\section{Acknowledgements}

Ellis Janzon has received grants (no 200704, 20080 I R) for this study by the Advisory Board of Tobacco Science, Sweden. There has been no collaboration with the sponsor during the study.

\section{References}

I. Critchley JA, Unal B: Health effects associated with smokeless tobacco: a systematic review. Thorax 2003, 58:435-443.

2. Critchley JA, Unal B: Is smokeless tobacco a risk factor for coronary heart disease? A systematic review of epidemiological studies. Eur J Cardiovasc Prev Rehabil 2004, 19:10I-II2.

3. Asplund K: Smokeless tobacco and cardiovascular disease. Prog Cardiovasc Dis 2003, 45:383-394.

4. Asplund K, Nasic S, Janlerts U, Stegmayr B: Smokeless tobacco as a possible risk factor for stroke in men - a nested case-control study. Stroke 2003, 34:1754-9.

5. Lee PN: Circulatory disease and smokeless tobacco in Western population: a review of the evidence. Int J Epidemiol 2007, 36:789-804.

6. Johansson S-E, Sundqvist K, Qvist J: Smokeless tobacco and coronary heart disease a I 2-years follow-up study. Eur J Cardiovasc Prev Rehabil 2005, I 2:387-92.

7. Hergens M-P, Ahlbom A, Andersson T, Pershagen G: Swedish moist snuff and myocardial infarction among men. Epidemiology 2005, 16:12-6.

8. Bolinder G, Alfredsson L, Englund A, De Faire U: Smokeless tobacco use and increased cardiovascular mortality among Swedish construction workers. Am J Public Health 1994, 84:399-404.

9. Bolinder G: Smokeless tobacco-a less harmful alternative? In The tobacco epidemic. Progress in Respiratory Research Volume 28. Edited by: Bolinger CT, Fagerström KO. Basel: Karger; 1997:199-212.

10. Haglund B, Eliasson M, Stenbeck M, Rosen M: Is moist snuff use associated with excess risk of IHD or stroke? A longitudinal follow-up of snuff users in Sweden. Scand J Public Health 2007, 35:618-22.

II. Hergens MP, Alfredsson L, Bolinder G, Lambe M, Pershagen G, Ye W: Long term use of moist snuff and the risk of myocardial infarction amongst men. J Intern Med 2007, 262:35I-359.

12. Huhtasaari F, Asplund K, Lundberg V, Stegmayr B, Wester PO: Tobacco and myocardial infarction: is snuff les dangerous than cigarettes? BMJ 1992, 305: I252-6.

13. Huhtasaari F, Lundberg V, Eliasson M, Janlert U, Asplund K: Smokeless tobacco as a possible risk factor for myocardial infarction: a population-based study in middle-aged men. J Am Coll Cardiol 1999, 34: 1784-90.

14. Berglund G, Elmståhl S, Janzon L, Larsson SA: The Malmö Diet and Cancer Study. Design and feasibility. J Intern Med 1993, 223:45-5I.

15. Manjer J, Elmståhl S, Janzon L, Berglund G: Invitation to a population-based cohort study: differences between subjects recruited using various strategies. Scand J Public Health 2002, 30:103-12.

16. Lindström M, Bexell A, Hansson BS, Isacsson S-O: Hur mår Malmö? The health situation in Malmö: a report from a mailed questionnaire 1994 in Swedish. Dept. of Community Medicine, Malmö University Hospital, Sweden; 1995. (In Swedish)

17. Zia E, Hedblad B, Pessah-Rasmussen H, Berglund G, Janzon L, Engström G: Blood pressure in relation to the incidence of cerebral infarction and intracerebral hemorrhage. Hypertensive hemorrhage: debated nomenclature is still relevant. Stroke 2007, 38:268I-5.

18. Rosevall M, Janzon L, Berglund G, Engström G, Hedblad B: Incidence of stroke is related to carotid IMT even in the absence of plaque. Atherosclerosis 2005, I 79:325-31.

19. World Health Organization: Definition and diagnosis of diabetes mellitus and intermediate hyperglycaemia: report of a WHO/IDF, consultation. Geneva, World Health Org; 2006.

20. Li C, Engströ M, Hedblad B, Berglund G, Janzon L: Risk of stroke and hormone replacement therapy. $A$ prospective cohort study. Maturitas 2006, 20(54): I I-18.

21. Statistics Sweden: Swedish Socioeconomic Classification (SEI). In Reports on Statistical Co-ordination Stockholm: Statistics Sweden; 1982:244-8.

22. Rosvall M, Östergren PO, Hedblad B, Isacsson SO, Janzon L, Berglund $\mathrm{G}$ : Occupational status, educational level, and the prevalence of carotid atherosclerosis in a general population sample of middle-aged Swedish men and women. Results from the Malmö Diet and Cancer Study. Am J Epidemiol 2000, 152:334-46.

23. Hedblad B, Jonsson S, Nilsson P, Engström G, Berglund G, Janzon L: Obesity and myocardial infarction - vulnerability related to 
occupational level and marital status. A 23-year follow-up of an urban male Swedish population. J Intern Med 2002, 252:542-550.

24. Anonymous: International Classification of Diseases. In Ninth revision (ICD-9) Geneva: Geneva: World Health Organization; 1977.

25. Causes of death 1995: The National Board of Health and Welfare. Center of Epidemiology. Stockholm, Sweden; 1997. ISBN 9I-720II88-2

26. Engström G, Jerntorp I, Pessah-Rasmussen H, Hedblad B, Berglund G, Janzon L: Geographic distribution of stroke incidence within an urban population: relations to socioeconomic circumstances and prevalence of cardiovascular risk factors. Stroke 200I, 32:1098-I03.

27. US Surgeon General: The health consequences of smoking. $A$ report of the Surgeon General 2004 [http://www.cdc.gov/tobacco/sgr/ sgr 2004/index.htm]. Atlanta, Georgia: US Department of Health and Human Services, Centre for Disease Control and Prevention, National Centre for Chronic Disease Prevention and Health Promotion, Office on Smoking and Health

28. International Agency for Research on Cancer: Tobacco smoke and involuntary smoking. Volume 83. Lyon, France: IARC; 2004 (IARC Monographs on the evaluation of carcinogenic risks for humans.)

29. Luo J, Zendehdel K, Adami H, Bofetta P, Nyren O: Oral use of Swedish moist snuff (snus) and risk for cancer of the mouth, lung, pancreas in male construction workers: a retrospective cohort study. Lancet 2007, 369:2015-20.

30. Ramström LM, Foulds J: Role of snus in the initiation and cessation of tobacco smoking in Sweden. Tob Control 2006, I5:210-14.

31. Eliasson M, Asplund K, Nasic S, Rodu B: Influence of smoking and snuff on the prevalence and incidence of type 2 diabetes amongst men: the northern Sweden MONICA study. J Intern Med 2004, 256:10I-10.

32. Rodu B, Stegmayr B, Nasic S, Coel P, Asplund K: The influence on smoking and smokeless tobacco use on weight amongst men. J Intern Med 2004, 255: I 02-7.

33. Gerend MA, Boyle RG, Peterson CB, Hatsukami DK: Eating behaviour and weight control among women using smokeless tobacco, cigarettes, and normal controls. Addictive Behaviou 1998, 23-24: $171-8$.

\section{Pre-publication history}

The pre-publication history for this paper can be accessed here:

http://www.biomedcentral.com/1471-2261/9/21/prepub
Publish with Bio Med Central and every scientist can read your work free of charge

"BioMed Central will be the most significant development for disseminating the results of biomedical research in our lifetime. "

Sir Paul Nurse, Cancer Research UK

Your research papers will be:

- available free of charge to the entire biomedical community

- peer reviewed and published immediately upon acceptance

- cited in PubMed and archived on PubMed Central

- yours - you keep the copyright
BioMedcentral 